\section{Guerrino Meneguzzi Daniel Aberdam Joëlle Vailly Jean-Paul Ortonne}

\title{
Vers la compréhension moléculaire des épidermolyses bulleuses héréditaires
}

Les épidermolyses bulleuses héréditaires sont caractérisées par l'apparition de clivages cutanés ("bulles") au niveau de la jonction dermo-épidermique. La fragilité des kératinocytes épidermiques basaux des patients souffrant d'épidermolyse bulleuse simple a été associée aux altérations des tonofilaments et à des mutations touchant les gènes des kératines 5 et 14 . Les épidermolyses bulleuses dystrophiques, comportant des anomalies des fibrilles d'ancrage de l'épiderme au derme, sont génétiquement liées au gène du collagène VII. Récemment, une protéine proche de la famille des laminines (la nicéine) a été impliquée comme étant la molécule candidate dans l'étiologie des épidermolyses bulleuses jonctionnelles, caractérisées par un décollement dermo-épidermique qui préserve les kératinocytes et les structures dermiques. L'identification des mutations génétiques induisant les différentes formes d'épidermolyses bulleuses héréditaires amèneront non seulement à une compréhension des mécanismes de cohésion de la peau, mais aussi à des diagnostics anténatals précoces et, peut-être, à de nouvelles approches thérapeutiques.

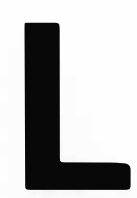

es épidermolyses bulleuses héréditaires $(\mathrm{EBH})$ sont des génodermatoses caractérisées cliniquement par une fragilité cutanée accrue conduisant, au moindre traumatisme, à la formation de clivages dans la peau (bulles) ; certaines variétés, comportant des atteintes extracutanées, sont létales. Toutes formes confondues, les épidermolyses bulleuses héréditaires atteignent environ un nouveau-né sur 50000 , avec une incidence dix fois moins élevée pour les formes les plus sévères. Selon des critères cliniques et morpho-histologiques, et selon le niveau de clivage dans la peau, on peut regrouper les épidermolyses bul- leuses héréditaires en trois grandes familles comprenant elles-mêmes plusieurs sous-types cliniques : les épidermolyses bulleuses épidermolytiques ou simples (EBS), associées à des bulles intra-épidermiques; les épidermolyses bulleuses jonctionnelles (EBJ), caractérisées par un clivage de la jonction dermo-épidermique au sein de la membrane basale, et les épidermolyses bulleuses dermolytiques ou dystrophiques (EBD), touchant le derme et présentant des clivages localisés immédiatement sous la membrane basale épidermique [1]. Récemment, des anomalies moléculaires spécifiques de certaines variétés d'EBH ont été décrites et des muta- 
tions génétiques impliquées dans des formes d'épidermolyse bulleuse simple et d'épidermolyse bulleuse dystrophique ont pu être identifiées. Ces avancées considérables ont été en partie réalisées grâce à une meilleure connaissance des structures moléculaires assurant la cohésion du derme et de l'épiderme.

\section{Kératines 5 et 14 et épidermolyses bulleuses simples}

Les épidermolyses bulleuses simples - les plus largement répandues des épidermolyses héréditaires - sont caractérisées par un décollement bulleux intra-épidermique résultant d'une cytolyse des kératinocytes de l'assise basale de l'épiderme sans altération de la jonction dermoépidermique. Cliniquement, ces génodermatoses, à transmission autosomique dominante, se présentent sous des formes variées. Les plus courantes sont représentées par l'épidermolyse bulleuse simple de type Weber-Cockayne, caractérisée par une éruption bulleuse localisée principalement sur les régions palmoplantaires, l'épidermolyse bulleuse simple de type Koebner, atteignant les mêmes zones du tégument que celles touchées par la forme WeberCockayne, et l'épidermolyse bulleuse simple de type Dowling-Meara, se traduisant par une éruption généralisée d'allure herpétiforme sévère avec évolution parfois létale.

L'implication des kératines dans la pathogénie des épidermolyses bulleuses simples avait été envisagée depuis les années 1980. En effet, à la suite d'observations ultrastructurales et immuno-histologiques menées chez des patients atteints d'épidermolyse bulleuse simple, il a été observé que l'épidermolyse bulleuse simple Koebner est associée à des modifications minimes du cytosquelette des kératinocytes, avec réduction ou condensation des tonofilaments*, et que l'épidermolyse bulleuse simple DowlingMeara est caractérisée par la présence d'agrégats intracytoplasmiques de tonofilaments. Récemment, l'implication des kératines de types 5 et 14 dans l'épidermolyse bulleuse simple Dowling-Meara, suggérée par des études immuno-histochimiques, a été confirmée par des études de transgé-

\footnotetext{
* Tonofilaments : filaments intracytoplasmiques présents dans les kératinocytes, résultant de l'organisation des molécules de kératine.
}

nèse montrant que des mutations spécifiques de ces molécules induisent la désorganisation du réseau des filaments intermédiaires des kératinocytes basaux et l'apparition d'un phénotype EBS chez les souriceaux nouveau-nés [2].

Ces deux kératines sont les constituants majeurs des filaments intermédiaires des kératinocytes de l'assise basale de l'épiderme. Chaque paire de molécules forme une unité hétérodimérique qui, par association antiparallèle avec un deuxième hétérodimère, donne origine à des structures tétramériques (protofilaments). Huit protofilaments enroulés en superhélice constituent un filament intermédiaire. Cette organisation des filaments est commune à toutes les cellules épithéliales, mais la paire de kératines qui constitue l'unité hétérodimérique de base varie selon les tissus et l'état de différenciation des kératinocytes. La formation des filaments, toutefois, résulte toujours de l'appariement spécifique d'une kératine de type acide (ou de type I; numérotées de 9 à 20) avec une kératine de type basique (ou de type II ; numérotées de 1 à 8 ). Les deux familles de kératines sont codées par des gènes localisés sur des chromoso-

\section{Tableau I}

\section{ANOMALIES MOLÉCULAIRES ASSOCIÉES AUX ÉPIDERMOLYSES BULLEUSES HÉRÉDITAIRES}

\begin{tabular}{|c|c|c|c|}
\hline Type clinique & Anomalie ultrastructurale & Protéines impliquées & $\begin{array}{l}\text { Localisation } \\
\text { chromosomique }\end{array}$ \\
\hline $\begin{array}{l}\text { Épidermolyse bulleuse simplex } \\
\text { Localisée (Weber-Cockayne) } \\
\text { Généralisée (Koebner) } \\
\text { Herpétiforme (Dowling-Meara) }\end{array}$ & $\begin{array}{l}\text { Tonofilaments } \\
\text { altérations du cytosquelette } \\
\text { altérations du cytosquelette } \\
\text { agrégats de tonofilaments }\end{array}$ & $\begin{array}{l}\text { Kératine } 5 \text { et kératine } 14 \\
\text { détectées } \\
\text { détectées } \\
\text { détectées }\end{array}$ & $12 q-17 q$ \\
\hline $\begin{array}{l}\text { Épidermolyse bulleuse dystrophique } \\
\text { Dominante: } \\
\text { Généralisée (Cockayne-Touraine) } \\
\text { Localisée } \\
\text { Récessive : } \\
\text { Généralisée mutilante (Hallopeau- } \\
\text { Siemens) } \\
\text { Généralisée non mutilante } \\
\text { Inversée } \\
\text { Localisée }\end{array}$ & $\begin{array}{l}\text { Fibrilles } d^{\prime} \text { ancrage } \\
\text { nombre réduit } \\
\text { nombre normal/réduit } \\
\text { absentes } \\
\text { nombre réduit } \\
\text { rudimentaires } \\
\text { nombre normal/réduit }\end{array}$ & $\begin{array}{l}\text { Collagène VII } \\
\text { normalement détecté } \\
\text { normalement détecté } \\
\text { non détecté } \\
\text { normalement détecté } \\
\text { normalement détecté } \\
\text { normalement détecté } \\
\text { ou diminué }\end{array}$ & $3 p 21$ \\
\hline $\begin{array}{l}\text { Épidermolyse bulleuse jonctionnelle } \\
\text { Généralisée grave (Herlitz) } \\
\text { Généralisée non grave (non } \\
\text { Herlitz) }\end{array}$ & $\begin{array}{l}\text { Hémidesmosomes } \\
\text { hypoplasiques, réduits ou } \\
\text { absents } \\
\text { hypoplasiques, réduits } \\
\text { ou absents }\end{array}$ & $\begin{array}{l}\text { Nicéine } \\
\text { non détectée ou très } \\
\text { diminuée } \\
\text { très diminuée ou } \\
\text { normalement détectée }\end{array}$ & \\
\hline
\end{tabular}


mes différents (le chromosome 17 (q12-21) pour le type I et le chromosome 12 (q11-13) pour le type II).

L'analyse fonctionnelle des différents domaines des kératines 5 et 14 par transfection de cellules épithéliales avec des ADNc mutés a permis d'établir que l'intégrité des régions amino- et carboxy-terminales du domaine central $\alpha$-hélicoïdal sont cruciales pour la formation du réseau de filaments de kératines in vivo et l'assemblage de filaments in vitro, et que celui-ci peut avoir lieu même si les domaines de la protéine flanquant le domaine central sont présents sur une seule des deux kératines de l'unité dimérique [3-6] (figure 1).

Les premières expériences de transgénèse concernant l'étude fonctionnelles des kératines ont été effectuées en utilisant un ADNc codant pour une kératine 14 tronquée dans l'extrémité C-terminale du domaine central. Il avait été démontré que cette construction, transfectée dans des kératinocytes en culture, induisait, même à des taux d'expression relativement faibles, une désorganisation du cytosquelette. Les souris transgéniques exprimant la mutation présentaient une extrême fragilité cutanée et, au moindre traumatisme, des décollements de la peau dus à une cytolyse des kératinocytes des assises profondes de l'épiderme. L'examen ultrastructural des tissus montrait la présence d'agrégats de tonofilaments dans le cytoplasme des kératinocytes basaux. Le phénotype était donc tout à fait superposable à celui des sujets atteints d'épidermolyse bulleuse simple Dowling-Meara. Les altérations du cytosquelette des kératinocytes de ces souris cultivés in vitro étaient aussi comparables à celles observées dans les kératinocytes humains transfectés avec le même ADNc. La corrélation entre l'intensité des altérations histologiques et le niveau d'expression du transgène confirmait la liaison de causalité entre la mutation et les effets observés. Afin d'établir si les variantes cliniques d'épidermolyses bulleuses simples pourraient être liées à des mutations affectant des régions différentes de cette kératine, de nouvelles séries de souris transgéniques ont été produites en utilisant les $\mathrm{ADNc}$ de kératine 14 délétés qui, transfectés dans des kératinocytes normaux en culture, altèrent les processus d'élongation et affectent la stabilité des filaments. De telles altérations ont été retrouvées dans les kératinocytes des souris transgéniques, qui, de plus, étaient sujets à des décollements épidermiques sur les coussinets plantaires. La similitude avec les lésions observées chez les patients souffrant d'épidermolyse bulleuse simple Weber-Cockayne ou Koebner était frappante. Sur la base de ces résultats, il a été conclu que des mutations de la kératine 14 pourraient être impliquées dans des variétés d'épidermolyse bulleuse simple dont l'aspect clinique dépendrait à la

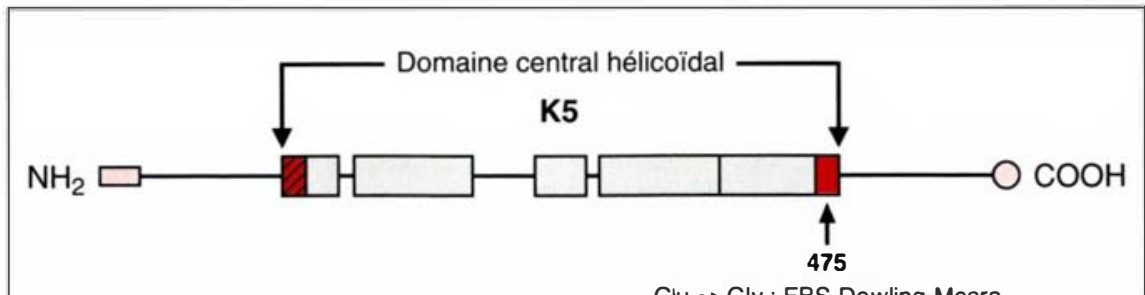

Glu $\rightarrow$ Gly : EBS Dowling-Meara

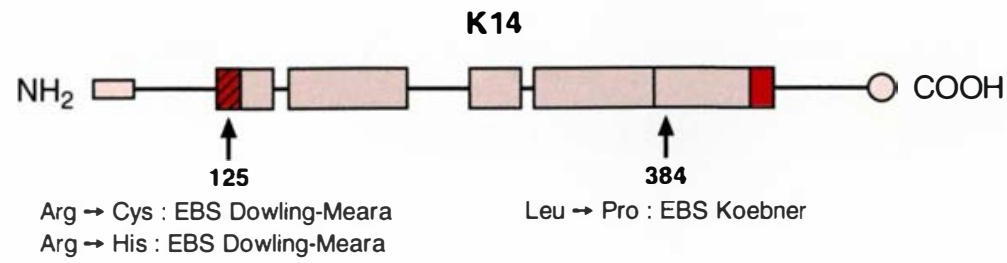

Figure 1. Représentation schématique des sites touchés par les mutations identifiées à ce jour sur les kératines 5 et 14. Chez les patients EBS, les séquences hautement conservées du domaine central hélicoïdal sont représentées en rouge hachuré et plein.

$\mathrm{m} / \mathrm{s} n^{\circ} 4$ vol. 9 , auril 93 fois de la nature de l'altération génétique et du niveau d'expression du gène muté.

Les régions de la kératine 14 essentielles au maintien de la structure du cytosquelette des kératinocytes basaux ayant été identifiées, il fallait vérifier que les mêmes régions de la molécule étaient mutées chez les patients atteints d'épidermolyse bulleuse simple. En effet, l'analyse par PCR de la région $\mathrm{N}$-terminale du domaine central de la kératine 14 pratiquée chez deux patients atteints d'épidermolyse bulleuse simple DowlingMeara a mis en évidence deux mutations ponctuelles distinctes touchant l'arginine 125. Dans un cas, le résidu est remplacé par une cystéine, dans l'autre par une histidine [4]. Ces mutations concernent un résidu issu d'une séquence d'acides aminés qui constitue le début de l' $\alpha$-hélice et qui est très conservée au cours de l'évolution des espèces, puisqu'elle est présente dans 47 des 51 protéines de filaments intermédiaires séquencées à ce jour. L'importance fonctionnelle de la portion $\mathrm{N}$-terminale du domaine central étant déjà amplement documentée, la reproduction de l'anomalie observée chez ces patients sur des cultures de kératinocytes a constitué la preuve définitive de l'implication du gène de la kératine 14 dans la pathogénie de l'affection. Par la même approche, dans une famille atteinte d'épidermolyse bulleuse simple Koebner, une épidermolyse bulleuse simple moins sévère que l'épidermolyse bulleuse simple DowlingMeara, une mutation ponctuelle a pu être localisée sur l'exon 6 du même gène, dans une région apparemment moins cruciale pour l'assemblage des filaments intermédiaires, comme il avait été montré auparavant par les travaux réalisés in vitro et sur les souris transgéniques. Cette mutation avait entraîné l'apparition d'un site de restriction $\mathrm{Mspl}$, qui était donc spécifique des sujets atteints. Le fait que ce polymorphisme n'ait pas été détecté chez 20 autres patients non apparentés atteints d'épidermolyse bulleuse simple a indirectement confirmé l'hypothèse selon laquelle toute une variété de mutations touchant au gène de la kératine 14 pourraient être impliquées dans l'étiologie des épidermolyses bulleuses simples [7]. 


\section{RÉFÉRENCES}

1. Fine JD, Bauer EA, Briggaman RA, Carter DM, Eady RAJ, Esterly NB, Holbrook KA, Hurwitz S, Johnson L, Lin A, Pearson R, Sybert VP. Revised clinical and laboratory criteria for subtypes of inherited epidermolysis bullosa. J Am Acad Dermatol $1991 ; 24: 119-35$

2. Vassar R, Coulombe PA, Degenstein L, Albers K, Fuchs E. Mutant keratin expression in transgenic mice causes marked abnormalities resembling human genetic skin disease. Cell 1991; 64: 365-80.

3. Coulombe PA, Chan YM, Albers K, Fuchs E. Deletions in epidermal keratins leading to alterations in filament organization in vivo and in intermediate filament assembly in vitro. $J$ Cell Biol 1990; 111 : 3049-64.

4. Coulombe PA, Hutton ME, Letai A, Hebert A, Paller AS, Fuchs E. Point mutations in human keratin 14 genes of epidermolysis bullosa simplex patients : genetic and functional analyses. Cell 1991; 66 : 1301-11.

5. Coulombe PA, Hutton ME, Vassar P, Fuchs E. A function for keratins and a common thread among different types of epidermolysis bullosa simplex diseases. $J$ Cell Biol 1991 ; 115 : 1661-74.

6. Wilson AK, Coulombe PA, Fuchs E. The roles of $\mathrm{K} 5$ and 14 head, tail, and $\mathrm{R} / \mathrm{KLLEGE}$ domains in keratin filament assembly in vitro. J Cell Biol $1992 ; 119$ : 401-14.

7. Bonifas JM, Rothman AL, Epstein EH Jr. Epidermolysis bullosa simplex : evidence in two families for keratin gene abnormalities. Science 1991 ; 254 : 1202-5.

8. Lane EB, Rugg EL, Navsaria $H$, Leigh IM, Heagerty AHM, IshidaYamamoto A, Eady RAF. A mutation in the conserved helix termination peptide of keratin 5 in hereditary skin blistering. Nature $1992 ; 356$ : 244-6.
Étant donné l'organisation moléculaire des filaments de kératines, résultant de l'appariement des kératines 5 et 14 , il était tout naturel de penser que des mutations affectant le gène de la kératine 5 pourraient aussi déterminer un phénotype EBS. Cette hypothèse a été vérifiée récemment par la détection d'anomalies de la kératine 5 chez des patients atteints d'épidermolyse bulleuse simple Dowling-Meara [8]. En effet, une étude électrophorétique des kératines a permis de mettre en évidence, chez deux sujets atteints, la coexistence d'une forme normale et d'une forme altérée de kératine 5. La molécule altérée, d'une taille apparente de $58 \mathrm{kDa}$ (au lieu de $59 \mathrm{kDa}$ ), n'est pas reconnue par un anticorps monoclonal réagissant avec la séquence consensus Thr-Tyr-Arg (Lys/Ser)-LeuLeu-Glu-Gly-Glu qui constitue le peptide de terminaison de l' $\alpha$-hélice du domaine hélicoïdal. Le séquençage du fragment d'ADN correspondant à cette région a révélé la présence d'une mutation ponctuelle du résidu 475 conduisant à la substitution d'un résidu Glu par un résidu Gly.

Ces résultats ont été confortés par les données des études de génétique de populations. Il y a plus de dix ans, l'association de l'épidermolyse bulleuse simple à une cassure du bras long du chromosome 12 avait déjà été rapportée. Plus récemment, l'étude d'une famille atteinte d'épidermolyse bulleuse simple Weber-Cokayne a permis de conclure que le locus lié à cette affection est situé sur le chromosome 12, à proximité du gène codant pour la kératine 5 [7], tandis que dans une autre famille atteinte d'épidermolyse bulleuse simple Weber-Cokayne, l'affection était manifestement liée aux marqueurs du chromosome 17 entourant le gène de la kératine 14 [9]. Dans un autre cas d'épidermolyse bulleuse simple, le chromosome 12 a été impliqué.

Il est maintenant établi que les épidermolyses bulleuses simples constituent un groupe d'affections dues à une variété de mutations touchant au moins deux gènes distincts (figure 1) et que la sévérité clinique de la maladie ne relève pas tant du fait qu'un gène plutôt que l'autre est lésé, mais de la localisation de la mutation et des conséquences fonctionnelles, plus ou moins délétères, qui lui sont associées. Étant donné que d'autres constituants cellulaires interviennent dans l'assemblage et l'organisation du cytosquelette du kératinocyte, il est possible que les différentes formes d'épidermolyse bulleuse simple ne soient pas toutes liées à des mutations des gènes des kératines 5 et 14 . En effet, une forte liaison génétique a été établie entre un sous-type rare d'épidermolyse bulleuse simple et le locus de la transaminase glutamo-pyruvique localisé sur le bras long du chromosome 8 et entre une épidermolyse bulleuse simple Koebner et le bras long du chromosome 1 .

\section{Collagène VII et épidermolyses bulleuses dystrophiques}

Les épidermolyses bulleuses dystrophiques sont caractérisées par un décollement bulleux superficiel localisé dans le derme, sous la lamina densa, et responsable de séquelles cicatricielles (grains de milium). Selon leur mode de transmission, ces affections ont été classées en deux groupes :

- Les épidermolyses bulleuses dystrophiques dominantes (EBDD), dont la forme principale, dite de CockayneTouraine, est caractérisée par une éruption bulleuse généralisée apparaissant à la naissance ou pendant la prime enfance, et épargnant, dans la majorité des cas, les muqueuses. L'examen ultrastructural montre une hypoplasie ou une absence de fibrilles d'ancrage.

- Les épidermolyses bulleuses dystrophiques récessives (EBDR) dont la forme classique, dite de HallopeauSiemens, se manifeste précocement par une éruption bulleuse généralisée, avec lésion des muqueuses qui entraîne des complications dentaires et des sténoses œesophagiennes responsables de malnutrition et de retards de croissance. La cicatrisation des érosions cutanées aboutit à des lésions atrophiques caractéristiques (syndactylies, contractures en flexion des membres) et à un décès précoce des patients. Dans une forme inversée, les bulles se localisent préférentiellement aux plis de flexion. Les marqueurs ultrastructuraux sont identiques à ceux des formes dominantes. 
Des observations au microscope électronique avaient permis d'établir une relation cntre des anomalies structurales des fibrilles d'ancrage et certaines formes d'épidermolyses bulleuses dystrophiques. Ultérieurement, il a été démontré que le constituant principal de ccs fibrilles d'ancrage est le collagène de type VII, si bien que cette protéine devint une excellente candidate pour expliquer la pathogénie de ces génodermatoses. Dans un premier temps, les manifestations pathologiques furent corrélées à un excès d'activité de la collagénase. En effet, une augmentation de la synthèse de collagénase avait été mise en évidence dans des cultures de fibroblastes de patients atteints d'épidermolyse bulleuse dystrophique récessive, et des résultats ultérieurs avaient suggéré que l'enzyme était structuralement anormale. Récem- ment, l'exclusion du gène de la collagénase, de la stromélysine-1, de la stromélysine-2 et de la collagénase interstitielle comme sites morbides associés à l'épidermolyse bulleuse dystrophique récessive ont rendu très improbable l'hypothèse imputant à une dégradation accrue du collagène les anomalies des fibrilles d'ancrage [10].

Dans la majorité des patients atteints d'EBDR Hallopeau-Siemens, le collagène VII n'est détectable ni par des techniques d'immunofluorescence sur des biopsies cutanées (figures $2 a$ et $b$ ), ni par Western blot à partir d'extraits dermiques et épidermiques. Ces observations concordent avec l'absence de fibrilles d'ancrage observée dans la peau des malades. Toutefois, l'expression intrakératinocytaire du collagène VII au cours de la cicatrisation cutanée chez des sujets atteints d'épidermolyse bulleuse dystrophique récessive a été récemment démontrée, ce qui suggère une capacité de ces cellules à synthétiser des molécules, peut-ĉtre incomplètes, de collagène VII. Les études par immunofluorescence soulignent l'hétérogénéité de ce groupe d'affections, puisque dans d'autres formes d'épidermolyse bulleuse dystrophique une immunoréactivité anticollagène VII est normalement détectée à la jonction dermo-épidermique.

La démonstration définitive de l'implication du collagène VII dans les épidermolyses bulleuses dystrophiques est très récente. Ce composant des épithéliums squameux stratifiés est une protéine homotrimérique Chaque chaîne qui la compose est synthétisée par les kératinocytes épidermiques et par les fibroblastes du derme sous forme d'un monomère, le
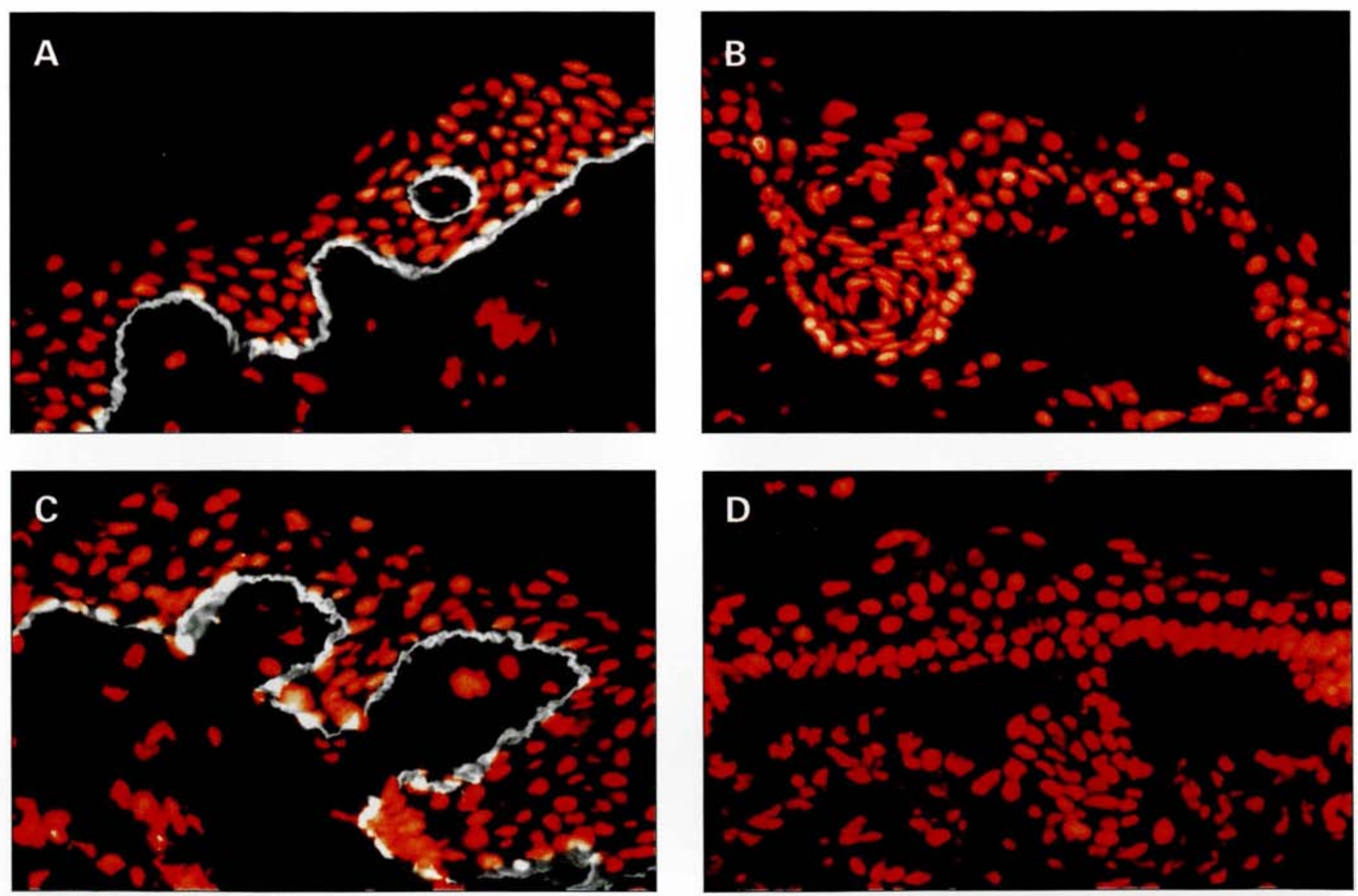

Figure 2. Immunofluorescence indirecte réalisée avec les anticorps monoclonaux LH-72 reconnaissant la molécule de collagène VII (A et B) et GB3 reconnaissant la molécule de nicéine (C et D) sur des biopsies cutanées de peau saine (A et $C$ ) ou atteinte d'épidermolyse bulleuse récessive dystrophique (B) et d'épidermolyse bulleuse jonctionnelle de type Herlitz (D). Le marquage linéaire de la jonction dermo-épidermique de la peau saine (A et C) n'est pas observé dans les peaux lésionnelles (B et D). Les noyaux cellulaires ont été colorés par l'iodure de propidium. 


\section{RÉFÉRENCES}

9. McKenna KE, Hughes AE, Bingham EA, Nevin NC. Linkage of epidermolysis bullosa simplex to keratin gene loci. $J$ Med Genet 1992 ; 29 : 568-70.

10. Hovnanian A, Dusquenoy $\mathrm{P}$, Anselem S, Blanchet-Bardon C, Lathrop $M$, Dubertret L, Goossens M. Exclusion of linkage between the collagenase gene and generalized recessive dystrophic epidermolysis bullosa phenotype. J Clin Invest 1991; 88 : 1716-21.

11. Parente MG, Chung LC, Ryynänen J, Woodley DT, Wynn KC, Bauer EA, Mattei MG, Chu ML, Uitto J. Human type VII collagen : cDNA cloning and chromosomal mapping of the gene. Proc Natl Acad Sci USA 1991; 88 : 6931-5.

12. Hovnanian A, Duquesnoy $\mathrm{P}$, BlanchetBardon C, Knowlton RG, Anselem S, Lathrop M, Dubertret L, Uitto J, Goossens M. Genetic linkage of recessive epidermolysis bullosa of the type VII collagen gene. J Clin Invest $1992 ; 90$ : 1033-7.

13. Ryynanen $M$, Knowlton RG, Parente MG, Chung LC, Chu ML, Uitto J. Human type VII collagen genetic linkage of the gene (COL7A 1 ) on chromosome 3 to dominant dystrophic epidermolysis bullosa. Am J Hum Genet 1991 ; 49 : 797-803.

14. Uitto J, Ryynanen M, Christiano AM, Hovnanian A, Frants R, Bauer EA, Knowlton RG. Genetic linkage of the type VII collagen gene (COL7A1) to dominant dystrophic epidermolysis bullosa (DDEB) in families with abnormal anchoring fibrils. $J$ Invest Dermatol 1992 ; 98 : 576A.

15. Epstein EH Jr. Molecular genetics of epidermolysis bullosa. Science 1992; 256 : 799-804.

16. Bruckner-Tuderman L. Evidence for a structural abnormality of collagen VII in a patient with dystrophic epidermolysis bullosa pro $\alpha 1(V I I)$, qui est constitué d'un long domaine collagénique d'environ $145 \mathrm{kDa}$ reliant deux domaines globulaires non collagéniques : le NC-1 (d'une taille approximative de $145 \mathrm{kDa})$ du côté aminoterminal, et le NC-2 (d'environ 30 kDa) à l'extrémité carboxylique. Une fois sécrétés, les monomères forment des dimères antiparallèles qui, après clivage protéolytique des domaines NC-2, s'agrègent latéralement et forment les fibrilles d'ancrage (figure 3). Celles-ci relient les molécules de collagène IV de la membrane basale dermoépidermique au collagène IV des plaques d'ancrage du derme. D'autres fibrilles, interagissant par les deux extrémités à la lamina densa, se croisent avec des fibres interstitielles constituées de collagène I et III, et contribuent ainsi à la formation du réseau qui permet l'adhérence de la membrane basale épidermique au tissu conjonctif dermique.

Les informations sur les propriétés et la structure du collagène VII obtenues par des études biochimiques ont été récemment complétées par le clonage d'ADNc correspondant à la quasi-totalité de la molécule. Grâce au criblage immunologique d'une banque d'expression construite à partir de kératinocytes humains avec des auto-anticorps anti-collagène VII produits par un patient souffrant d'épidermolyse bulleuse acquise, un ADNc codant pour la partie carboxyterminale du domaine collagénique et le domaine NC-1 a été isolé [11]. La séquence primaire déduite de l'analyse des ADNc a permis, d'une part, d'établir la taille et la position exacte des domaines NC-1 et NC-2 (les études biochimiques avaient placé NC-1 du côté C-terminal de la protéine) et, d'autre part, de définir l'organisation de la protéine.

Par hybridation in situ, grâce aux sondes $\mathrm{ADNc}$, le gène du collagène VII (COL7A1) a été localisé par la suite sur le bras court du chromosome 3, dans le locus 3p21. D'une longueur d'environ $25 \mathrm{~kb}$, COL7A1 est constitué de plus de 140 exons. L'analyse de la structure du gène et l'identification des motifs retrouvés dans les molécules d'adhésion, notamment deux tripeptidiques potentiels d'adhérence RGD, constituent des éléments en faveur de l'hypothèse qui assigne à NC-1 une

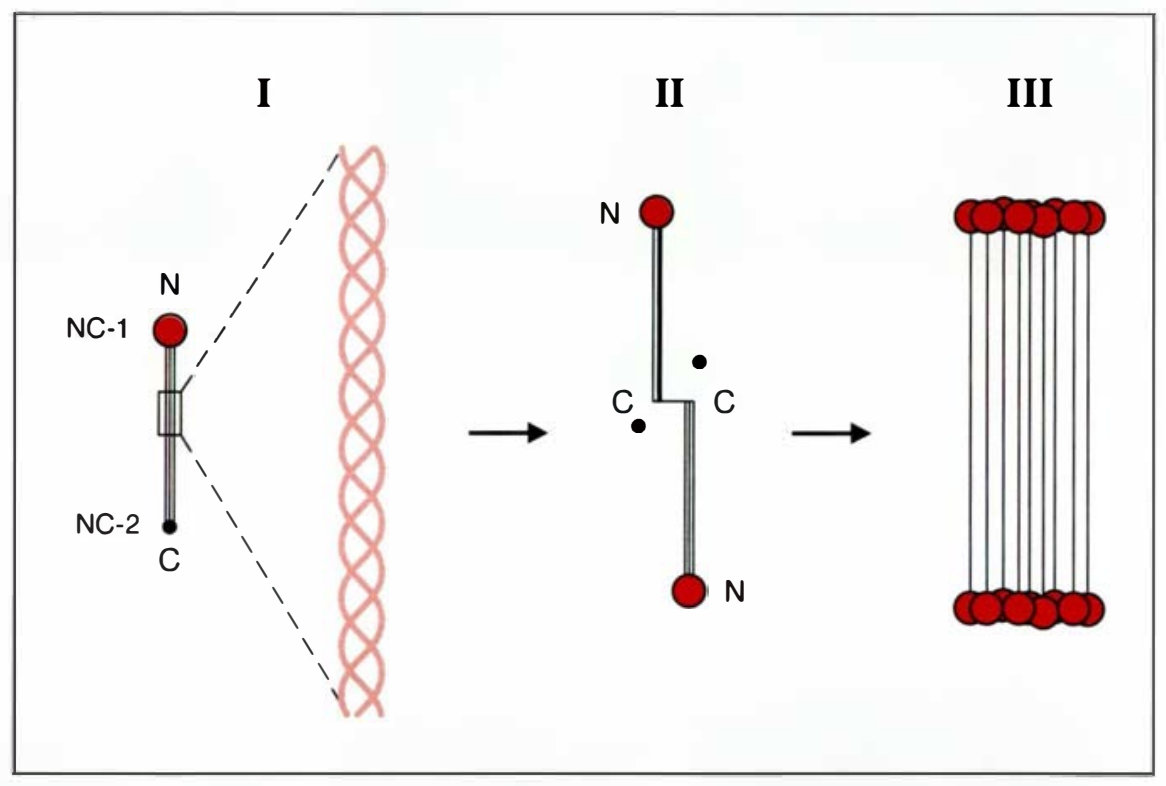

Figure 3. Diagramme représentant la structure du procollagène de type VII et son assemblage dans les fibrilles d'ancrage. Le procollagène est synthétisé sous forme d'une molécule homotrimérique constituée d'un domaine central hélicoïdal flanqué de deux domaines globulaires NC-1 et NC-2 (I). Deux molécules de procollagène s'associent par la formation de ponts disulfures au niveau de la région C-terminale et les domaines NC-2 sont perdus par clivage protéolytique (II). Les dimères s'associent côte à côte pour former les fibrilles d'ancrage (III). $N$ : extrémité amino-terminale; $C$ : extrémité carboxy-terminale. 
fonction importante dans l'interaction des fibrilles d'ancrage avec les composants de la membrane basale et des plaques d'ancrage.

Les études de liaison génétique ont directement impliqué COL7A1 dans l'étiologie de l'épidermolyse bulleuse dystrophique [12-14]. Initialement, l'analyse par Southern blot de l'ADN d'individus sains non apparentés a conduit à l'identification d'un polymorphisme de restriction (RFLP*) dans les produits de digestion par l'endonucléase PvuII. Il fut ainsi possible de démontrer le lien entre COL7A1 et le phénotype pathologique dans des familles affectées par la forme dominante d'épidermolyse bulleuse dystrophique. Ce résultat a été confirmé sur un échantillon élargi de familles et par l'étude de la ségrégation de deux marqueurs flanquant le locus COL7A1 sur le bras court du chromosome 3. En effet, le locus morbide apparaît lié au locus COL7 A1, avec un lod-score supérieur à 27 et une fréquence de recombinaison $\theta=0$. De plus, l'étude des RFLP étendue à 24 familles présentant des cas d'épidermolyse bulleuse dystrophique récessive de type Hallopeau-Siemens a démontré que, dans 19 d'entre elles, la transmission de la maladie est aussi liée au locus COL7 A1 avec un lod-score de 3,97 avec $\theta=0$. Un deuxième RFLP reconnu par l'endonucléase Alu I a été récemment décrit. L'ensemble des données confirme donc l'hypothèse, fondée sur les observations de microscopie électronique, selon laquelle les épidermolyses bulleuses dystrophiques sont corrélées à des anomalies des fibrilles d'ancrage dues à des altérations du collagène VII. Grâce à ces progrès, un diagnostic anténatal d'épidermolyse bulleuse dystrophique récessive de type Hallopeau-Siemens a pu être réalisé récemment à partir d'une biopsie de trophoblaste (prélevée à 12 semaines d'aménorrhée) sur une femme ayant des enfants atteints et d'autres indemnes de l'affection. Le diagnostic d'exclusion, fondé sur l'utilisation du RFLP Pvu II, a été confirmé par la naissance d'un nouveauné indemne d'épidermolyse bulleuse dystrophique récessive (A. Hovnanian, communication personnelle).

\footnotetext{
* RFLP : restriction fragment length polymorphism.
} $\mathrm{m} / \mathrm{s} n^{\circ} 4$ vol. 9, avril 93
Il est fort probable que toute une variété de mutations touchant à la synthèse ou à la maturation du collagène de type VII sont à l'origine des différentes formes cliniques d'épidermolyse bulleuse dystrophique [15]. Ainsi, dans la forme d'épidermolyse bulleuse dystrophique inversée, le collagène VII est normalement détecté à la jonction dermo-épidermique ; toutefois les fibrilles d'ancrage présentent un aspect rudimentaire. Chez un sujet atteint, des molécules de collagène de type VII ont été détectées par Western blot dans les extraits dermiques et épidermiques suggérant que les fibrilles d'ancrage sont constituées de dimères instables de collagène [16]. Par ailleurs, une rétention du collagène VII dans les kératinocytes de l'assise basale, associée à une diminution de l'immunoréactivité de la jonction dermo-épidermique, a été observée par immunofluorescence indirecte chez plusieurs nouveau-nés présentant soit un tableau clinique d'épidermolyse bulleuse dystrophique, soit un tableau de dermatose bulleuse transitoire du nouveau-né. Cette anomalie, pour laquelle le collagène de type VII est impliqué, mérite d'être mieux caractérisée grâce à une analyse élargie à d'autres familles.

\section{Nicéine et épidermolyses bulleuses jonctionnelles}

Les épidermolyses bulleuses jonctionnelles sont transmises sur un mode récessif et se caractérisent par une éruption bulleuse superficielle qui, en guérissant, ne laisse pas de cicatrices. L'examen ultrastructural montre un clivage dans la lamina lucida associé à des anomalies plus ou moins sévères des hémidesmosomes et de la plaque dense sous-hémidesmosomiale* . Ces génodermatoses constituent un groupe hétérogène représenté par deux formes principales : l'épidermolyse bulleuse jonctionnelle Herlitz et l'épidermolyse bulleuse jonctionnelle non Herlitz. L'épidermolyse bulleuse jonctionnelle Herlitz (ou gravis) se manifeste dès la naissance par l'apparition de bulles cutanées étendues et par des atteintes aux muqueuses (laryngée,

\footnotetext{
- Pour le rappel de la structure de la jonction dermoépidermique, voir l'article de J.-F. Nicolas, et al., p. 376 de ce numéro.
}

digestive, respiratoire) responsables de complications sévères; le décès survient habituellement avant la deuxième année, par asphyxie ou surinfection avec septicémie. L'épidermolyse bulleuse jonctionnelle non Herlitz se caractérise par une évolution prolongée, jusqu'à l'âge adulte. Des formes localisées ou inversées, caractérisées par les mêmes anomalies ultrastructurales que les formes Herlitz, ont également été décrites. Grâce à l'anticorps monoclonal GB3, obtenu par immunisation avec des cellules amniotiques humaines, notre laboratoire a mis en évidence une anomalie de la jonction dermoépidermique des patients atteints d'épidermolyse bulleuse jonctionnelle Herlitz. L'anticorps réagit intensément avec la jonction dermoépidermique des peaux saines et des annexes épithéliales, mais, dans la grande majorité des sujets atteints d'épidermolyse bulleuse jonctionnelle Herlitz, le marquage n'est pas détecté (figures $2 c$ et $d$ ). Cette absence spécifique est observée dans toutes les membranes basales (épithélium digestif, respiratoire, vésical, amniotique...) où l'antigène reconnu par GB3 est normalement présent dès la $8^{\mathrm{e}}$ semaine de gestation [17].

Par l'étude immunohistochimique de cellules en culture, nous avons démontré que l'antigène est synthétisé et sécrété par les kératinocytes, normaux ou transformés, mais qu'il n'est pas décelable dans le cas de cellules provenant d'explants de patients EBJ Herlitz. Puisque ces derniers présentent une moindre capacité d'adhérence et expriment normalement tous les constituants connus de la jonction dermo-épidermique, il est concevable que les épidermolyses bulleuses jonctionnelles, ou tout au moins les formes les plus graves, soient associées à un défaut génétique impliquant cette molécule spécifiquement synthétisée par les kératinocytes [17].

L'ensemble de ces observations nous a conduits, d'une part, à l'isolement et à la caractérisation de cet antigène et, d'autre part, à l'utilisation de l'anticorps dans le diagnostic rapide et anténatal de l'épidermolyse bulleuse jonctionnelle Herlitz par immunofluorescence indirecte sur des biopsies de peau fœtale à 20-21 semaines 


\section{RÉFÉRENCES}

17. Verrando $P$, Blanchet-Bardon C Pisani A, Thomas L, Cambazard F, Eady RAJ, Schofield O, Ortonnc JP. Monoclonal antibody GB3 defines a widespread defect of several basement membranes and a keratinocyte dysfunction in patient with lethal junctional epidermolysis bullosa. Lab Invest $1991 ; 64: 85-92$

18. Schofield OMV, Fine JD, Verrando $P$ Heagerty AHM, Ortonnc JP, Eady RAJ GB3 monoclonal antibody for the diagnosis of junctional cpidermolysis bullosa : results of a multicenter study. $J$ Am Acad Dermatol $1990 ; 123$ : 1078-83.

19. Verrando P, Pisani A, Ortonne JP. The new basement membranc antigen recognized by the monoclonal antibody GB3 is a large sizc glycoprotein : modulation of its expression by retinoic acid. Biochim Biophys Acta 1988 ; 942 : 45-56.

20. Baudoin C, Galliano MF, Verrando P, Ortonne JP, Meneguzzi G. Caracterization of nicein $150 \mathrm{kDa}$ cDNAs reveals homologies with human laminin A chain. J Invest Dermatol 1993 (sous presse).

21. Verrando $P$, Vailly J, Champliaud MF, Gerecke D, Wagman W, Baudoin C, Abcrdam D, Burgeson R, Bauer E, Ortonne JP. A new laminin variant defined by the cDNA scquence of the $100 \mathrm{kDa}$ subunit of the basement membrane protein nicein (BM-600) kalinin. J Biol Chem 1993 (sous presse).

22. Verrando P, Pisani A, Hsi BL, Yeh CJ, Scricys N, Ortonnc JP. Monoclonal antibody GB3, a new tool for the study of human basement membranes and hemidesmosomes. Exp Cell Res 1987; 170 : 116-28.

23. Meneguzzi G, Marinkovich MP, Aberdam D, Pisani A, Burgeson R, Ortonne $\mathrm{JP}$. Kalinin is abnormally expressed in epithelial basement membranes of Herlitz's junctional epidermolysis bullosa patients. Exp Dermatol 1993 (sous pressc).

24. Marinkovich MP, Verrando P, Keene DR, Meneguzzi G, Lunstrum GP, Ortonne J-P, Burgeson RE. The basement membranc proteins kalinin and nicein are structurally and immunologically homolo- de gestation. En effet, une expression normale de la molécule reconnue par GB3 exclut cette affection [18].

L'antigène reconnu par GB3, que nous avons baptisé nicéine, a été identifié comme étant une sialoglycoprotéine d'environ $600 \mathrm{kDa}$, constituée de trois sous-unités (de 100, 125 et $150 \mathrm{kDa}$ ), et localisée dans la partie profonde de la lamina lucida, au contact de la lamina densa [19, 20]. Elle est présente sous la plaque sousbasale, et plus rarement entre les hémidesmosomes. La purification de la nicéine par chromatographie d'affinité à partir de milieux de culture conditionnés par des kératinocytes et l'obtention d'anticorps polyclonaux dirigés contre chacune de ses sousunités ont permis l'isolement des $\mathrm{ADNc}$ correspondants. La séquence nucléotidique des clones codant pour la sous-unité de $100 \mathrm{kDa}$ nous a révélé que la nicéine est une protéine apparentée à la famille des laminines [20]. En effet, l'extrémité Nterminale du polypeptide possède une homologie importante avec le bras court de la chaîne B2 de la laminine humaine et, comme celui-ci, présente une organisation de type EGF-like propre aux protéines possédant de multiples domaines fonctionnels. Les régions C-terminales des deux protéines sont aussi homologues, bien qu'à un moindre degré. La prédiction de la structure secondaire révèle le même type de conformation, notamment des tours $\beta$ dans les bras courts et des hélices $\alpha$ interrompues dans les bras longs. La parenté des deux protéines est confirmée par la localisation chromosomique des ADNc, établie sur le chromosome 1 en q25-q31, à proximité du locus codant pour la chaîne B2 de laminine. A ce jour, les données obtenues par le séquençage des ADNc codant pour la sous-unité $125 \mathrm{kDa}$ et $150 \mathrm{kDa}$ vont dans le même sens. Des homologies significatives ont été décelées entre la région carboxy-terminale du polypeptide $150 \mathrm{kDa}$ et la région globulaire en 3' de la chaîne A de la laminine, comprenant une structure tripeptidique potentielle d'adhérence (RGD) [21]. La localisation chromosomique en $18 \mathrm{q} 11.2$ du gène codant pour cette sous-unité est distincte de celle de la chaîne A (18p11.3), mais, étant donné que chez l'homme le chromo- some 18 a subi une inversion péricentrique absente chez les primates, ces résultats confirment l'hypothèse selon laquelle la nicéine et la laminine appartiennent à deux familles distinctes de gènes apparentés, ayant divergé à partir d'un ancêtre commun au cours de l'évolution. Les différentes sondes reconnaissent des ARNm spécifiques, toutefois les ADNc codant pour la sous-unité de $150 \mathrm{kDa}$ hybrident aussi avec les ARNm correspondant à la chaîne $A$ de la laminine humaine, ce qui pourrait indiquer que des séquences fonctionnellement importantes sont préservées. Toutefois, l'utilisation d'épidermes humains reconstitués in vitro et exposés à l'acide rétinoïque nous a permis de montrer que l'expression de la nicéine n'est pas soumise à la même régulation que celle de la laminine [22].

Vraisemblablement, les termes EBJ "Herlitz " et " non Herlitz" recouvrent une variété de mutations touchant les trois gènes de la nicéine. A l'heure actuelle, il n'est pas possible d'exclure que d'autres molécules soient impliquées, même indirectement, dans l'étiologie des épidermolyses bulleuses jonctionnelles. En effet, un anticorps monoclonal, le 19-DEJ-1, qui réagit avec un antigène du complexe hémidesmosomefilament d'ancrage, n'est pas immunoréactif avec la jonction dermoépidermique de la peau d'un certain nombre des patients EBJ. Cet anticorps ne reconnaît pas la nicéine et son antigène n'est pas caractérisé. Cependant, cet antigène est également altéré au cours de l'épidermolyse bulleuse dystrophique récessive (dans environ $50 \%$ des cas), si bien qu'il n'est pas encore clairement établi si le 19-DEJ-1 révèle un épiphénomène secondaire à l'anomalie primitive des épidermolyses bulleuses jonctionnelles ou bien s'il est l'indication d'un polymorphisme étiologique intéressant plusieurs types de molécules d'adhérence. Par ailleurs, la nicéine paraît très proche de deux autres molécules récemment décrites : la kalinine et l'épiligrine. En effet, le nombre et la taille des sous-unités, ainsi que la réactivité croisée des anticorps dirigés contre chacune de ces protéines suggèrent qu'elles pourraient correspondre au même compo- 
sant de la membrane basale. Nous avons montré que, comme la nicéine, la kalinine n'est pas détectée par immunofluorescence dans les tissus de patients EBJ [23], que ces deux protéines ont le même profil par les analyses biochimiques de produits de clivage par protéase V8 [24] et les mêmes séquences d'ADNc correspondant aux sous-unités de $100 \mathrm{kDa}[21]$. Cette identité ne pourra être définitivement affirmée que lorsque des séquences seront disponibles pour les autres sous-unités de ces protéines. Les analyses des RFLP et le typage génotypique des échantillons d'ADN des familles des patients, que nous avons entrepris, devraient bientôt fournir les données d'exclusion allélique et de déséquilibre de liaisons permettant d'identifier les gènes impliqués dans les épidermolyses bulleuses jonctionnelles et de procéder à la recherche d'éventuelles mutations.

En ce qui concerne le dépistage prénatal des épidermolyses bulleuses jonctionnelles, à très court terme, l'objectif principal est constitué par la mise au point d'un test moins traumatisant que la biopsie fotale, une technique invasive qui ne peut être réalisée que dans quelques centres spécialisés. La mise en évidence de la nicéine dans le liquide amniotique pourra servir de base à une nouvelle approche, moins agressive, réalisée à partir de liquide amniotique prélevé par amniocentèse. Toutefois, l'avenir du diagnostic des épidermolyses bulleuses jonctionnelles se situe dans la mise au point de techniques de génétique moléculaire utilisant des échantillons d'ADN extrait de biopsies de trophoblaste, un procédé plus simple et plus sûr permettant un diagnostic très précoce.

Comme le montrent ces résultats récents, la génétique moléculaire fait entrer l'histoire des épidermolyses bulleuses héréditaires dans une ère nouvelle. L'étude de nouvelles familles souffrant de ces affections permettra d'identifier les mutations les plus fréquemment impliquées dans les séquences décrites ci-dessus. Plus généralement, la mise en évidence de telles mutations permettra de lever les difficultés de classification de ces génodermatoses, cliniquement nombreuses et hétérogènes. Cela s'expli$m / s n^{\circ} 4$ vol. 9, avril 93 que par le fait que la cohésion dermo-épidermique est le résultat d'interactions moléculaires complexes, et que les structures fines du cytosquelette des kératinocytes, des hémidesmosomes et des molécules d'ancrage dermo-épidermiques sont mal connues. De plus, il est possible que d'autres molécules, encore non identifiées, soient également impliquées dans la pathogénie de l'épidermolyse bulleuse jonctionnelle

\section{Summary}

Recent insights into the molecular bases of hereditary epidermolysis bullosa

Hereditary epidermolysis bullosa (EB) are clinically heterogeneous disorders characterized by blistering and erosions of the epidermis in response to trauma. The molecular basis underlying the main forms of these diseases have been recently brought to light. The simplex types (EBS), characterized by blister formation within the epidermis at the level of basal keratinocytes, have been associated with specific mutations of keratins 5 and 14 leading to an impaired assembly of tonofilaments and fragility of keratinocytes. The dystrophic EB (DEB), where cleavage occurs within the upper dermis at the level of anchoring fibrils, have been genetically linked to the type VII collagen locus, with no evidence for genetic heterogeneity thus far. This progress has been made possible by the identification of a polymorphism within the recently cloned human collagen VII gene. In junctional EB (JEB), characterized by dermal-epidermal splitting occurring within the lamina lucida, the causative defect appears related to the expression of a laminin-like protein named nicein, localized to anchoring filaments of the hemidesmosomes. Molecular cloning of the nicein genes is employed to identify putative mutations underlying the different forms of this affection. The application of molecular genetic to study inherited disorders of the skin is likely to lead soon to new classifications of cutaneous diseases, a prerequisite for perfecting any future therapeutic approach.

\section{Remerciements}

Nous remercions Anne Pisani pour sa participation aux études immunomorphologiques. 